\title{
ANÁLISIS DE LA PRESENCIA DE VIOLENCIA INSTITUCIONAL EN EL CONTEXTO ESCOLAR EN EL NIVEL MEDIO SUPERIOR
}

\section{ANALYSIS OF THE PRESENCE OF INSTITUTIONAL VIOLENCE IN THE SCHOOL CONTEXT AT THE HIGHER MIDDLE LEVEL}

Luz Alejandra Escalera Silva ${ }^{1}$

Sandra Rubí Amador Corral ${ }^{2}$

Luz Amparo Silva Morín ${ }^{3}$

Universidad Autónoma de Nuevo León

\footnotetext{
$1 \quad$ Luz Alejandra Escalera Silva. Doctora en Métodos Alternos de Solución de Conflictos por la Universidad Autónoma de Nuevo León. Profesora de la Facultad de Trabajo Social y Desarrollo Humano, Universidad Autónoma de Nuevo León, México. Correo electrónico: luz.escaleraslv@ uanl.edu.mx Orcid ID: https://orcid.org/0000-0002-3214$\underline{2834}$

2 Sandra Rubí Amador Corral: Doctora en Filosofía con Orientación en Trabajo Social y Políticas sociales de Bienestar Social por la Universidad Autónoma de Nuevo León. Profesora de la Facultad de Trabajo Social y Desarrollo Humano, Universidad Autónoma de Nuevo León, México. Correo electrónico: sandra.amadorcr@uanl.edu.mx Orcid ID: https://orcid.org/0000-0002-4814-6071 3 Luz Amparo Silva Morín: Maestría en Trabajo Social por la Universidad Autónoma de Nuevo León, Profesora de la Facultad de Trabajo Social y Desarrollo Humano, Universidad Autónoma de Nuevo León, México.

Correo electrónico: luz.silvamr@uanl.edu.mx

Orcid ID: https://orcid.org/0000-0003-0987-7557
}

\section{RESUMEN}

En la actualidad la violencia escolar se encuentra como una de las principales problemáticas que aquejan al sistema educativo en todos sus niveles. En los últimos años ha recibido una atención especial dado que ha puesto de manifiesto que escuelas e instituciones, no están exentas del entorno conflictivo que ha permeado en la sociedad mexicana desde hace algunos años. A pesar del creciente abordaje del fenómeno de violencia entre iguales, conocido como bullying en la literatura académica, pocos 
estudios se han interesado específicamente en la violencia institucional, misma que es ejercida en el plantel escolar por autoridades y docentes hacia los estudiantes, y sigue vigente no obstante los esfuerzos legislativos de las autoridades mexicanas para garantizar espacios educativos libres de violencia. Abordar los diferentes tipos de violencia que existen desde la ya muy conocida violencia entre iguales hasta la violencia institucional en el contexto académico y sus consecuencias en las personas que intervienen resulta necesario en un contexto social en el que las políticas educativas desarrolladas por las autoridades para combatir prácticas de violencia escolar han tenido resultados limitados. El presente estudio tiene como objetivo analizar a presencia de violencia escolar, sus tipos y principales consecuencias en una preparatoria publica del área metropolitana de Monterrey, N.L.

PALABRAS CLAVE: violencia escolar, bullying, violencia institucional, hostigamiento escolar, educación media superior.

\section{ABSTRACT}

Currently, school violence is one of the main problems that afflict the educational system at all levels. In recent years, it has received special attention, since it has shown that schools and institutions are not exempt from the conflictive environment that has permeated Mexican society for some years. Despite the growing attention to the phenomenon of violence between equals, known as bullying in academic literature, few studies have been specifically interested in institutional violence, which is exercised on school premises by authorities and teachers towards students, and continues to be in force despite the legislative efforts of the Mexican authorities to guarantee educational spaces free of violence. Addressing the different types of violence that exist from the already well-known violence between equals to institutional violence in the academic context and its consequences on the people involved is necessary in a social context where educational policies developed by the authorities to combat school violence practices have had limited results. The present study aims to analyze the presence of school violence, its types and main consequences in a public high school in the metropolitan area of Monterrey, N.L.

KEYWORDS: school violence, bullying, institutional violence, school harassment, highschool education.

\section{INTRODUCCIÓN}

\section{La violencia presente en las relaciones sociales que coexisten en el centro educativo.}

Para Del Tronco \& Madrigal (2017) al hablar de violencia escolar debemos tomar en cuenta sus dimensiones, es decir, las relaciones sociales que coexisten dentro del centro educativo, así como él marco institucional y normativo que previene o sanciona la violencia escolar.

La violencia entre iguales es la más conocida debido a la posición vulnerable que enfrenta el estudiante que juega el papel de víctima frente al victimario, siendo el bullying la práctica más común, mismo que se define como la agresión intencional reiterada de un estudiante a otro con la intención de generar daño y temor (Olewus, 1998) y se ha presentado entre los alumnos en diferentes tipos de manifestaciones, por una parte está el bullying físico que comprende daño corporal como golpes, empujones, estirones de cabello, siendo ésta la manifestación de violencia más fácil de identificar por dejar huellas corporales (Estevez, 2005; Cobo \& Tello, 2008).

Asimismo, podemos mencionar el bullying gesticular que refiere hacer gestos o señas a las víctimas con la finalidad de incomodarlas o amenazarlas; por último, señalamos el bullying verbal consistente en apodar, insultar, denigrar o difundir rumores con el objetivo de humillar a 
la víctima, siendo éste tipo el más frecuente en el ambiente escolar (Harries, 2003; Olewus D. , 2012).

Además, la violencia entre iguales no para en el espacio físico, también la encontramos en el espacio cibernético a través del Ciberbullying que es la práctica de la agresión adaptada a la cultura digital, en la que el agresor de manera intencional utiliza plataformas electrónicas de contacto, en repetidas ocasiones para intimidar a la víctima apoyándose de las Tecnologías de la Comunicación y la Información (TIC) e internet (Escalera, 2018).

Autores como Escalera (2018) hacen mención a las consecuencias que resultan de una práctica de violencia escolar como bullying tanto para la víctima como para el victimario, por ejemplo, mientras que en la víctima las consecuencias de la agresión impactan en el autoestima y el desequilibrio emocional, en el victimario se observa cómo al asumir un rol principal en el juego de poder, se empodera.

En palabras de Ortega (2008) la violencia escolar es entendida como un modelo de relación desigual entre aquellos de los que se espera una relación igualitaria, es decir, este concepto se enfoca exclusivamente en las relaciones entre estudiantes, excluyendo otras violencias que pudiesen presentarse en el centro educativo, por ejemplo, la violencia institucional en el contexto escolar que es el ejercicio de la desigualdad y el abuso de poder de un docente o autoridad educativa frente al estudiante, una práctica que también constituye violencia escolar.

Entre las manifestaciones de violencia institucional en el contexto escolar, podemos destacar el hostigamiento académico, que hemos definido como el ejercicio de poder del maestro frente al estudiante para humillarlo, prohibir el acceso a las instalaciones o discriminarlo hasta manipular calificaciones con el objetivo de intimidarlo, avasallarlo o perjudicarlo solo o en compañía de otros estudiantes (Barrientos, 2018).

A pesar de que estas prácticas son cada vez más frecuentes en los planteles educativos, tanto los investigadores de las ciencias sociales como las autoridades legislativas han limitado su estudio a la violencia escolar entre iguales, dejando fuera la violencia institucional derivada en el hostigamiento académico.

\section{Violencia en el contexto escolar. Los esfuerzos normativos para prevenirla, atenderla y erradicarla.}

La Organización de las Naciones Unidas para la Educación, la Ciencia y la Cultura (UNESCO, 2019), en su informe Behind the numbers: Ending school violence and bullying manifestó la problemática de la violencia escolar como una cuestión relevante para todos los países del mundo; según este reporte $48.2 \%$ de estudiantes de 144 países padecen o han padecido acoso dentro y fuera de sus centros de estudio.

La violencia en el plantel educativo se ha incrementado de manera acelerada obligando a las autoridades a esbozar esfuerzos legislativos por entidad federativa para dar respuesta al fenómeno de violencia en los centros educativos y proteger a los niños, niñas y adolescentes en el contexto escolar (Escalera, Silva, \& Contreras, 2019: 97).

La Ley General de Educación establece obligatoriedad para todas las instituciones educativas para aplicar métodos para prevenir y eliminar cualquier forma de violencia, sin embargo, a diferencia de las legislaciones específicas en la materia, no hace referencia a los diferentes tipos de violencia escolar, como acoso escolar o el hostigamiento académico. De hecho, las legislaciones estatales en materia de violencia escolar sólo reconocen la violencia 
entre iguales y usan indistintamente diferentes términos como sinónimos bullying. (Véase tabla

1).

\section{TABLA 1. TÉRMINOS DE MALTRATO ENTRE IGUALES CONTEMPLADOS EN LAS LEGISLACIONES EN MATERIA DE VIOLENCIA ESCOLAR.}

\begin{tabular}{|c|c|}
\hline Estados & Términos \\
\hline $\begin{array}{l}\text { Aguascalientes, Baja California Sur, Colima, } \\
\text { Guanajuato, Guerrero, Hidalgo, Michoacán, } \\
\text { Nuevo León, Oaxaca, Puebla, Quintana Roo, } \\
\text { Sinaloa, Tamaulipas, Tlaxcala, Veracruz y } \\
\text { Yucatán }\end{array}$ & Violencia Escolar \\
\hline $\begin{array}{l}\text { En Baja California, Coahuila, Colima, Nuevo } \\
\text { León, Oaxaca Puebla, Veracruz y Zacatecas }\end{array}$ & Acoso Escolar \\
\hline $\begin{array}{l}\text { En Baja California Sur, Ciudad de México, } \\
\text { Guerrero, Colima y Puebla }\end{array}$ & Hostigamiento \\
\hline
\end{tabular}

(Escalera, Silva, \& Contreras, 2019: 114).

Por otra parte, en la revisión de leyes estatales pudimos constatar que solo veinticuatro Estados cuentan con una ley específica contra la violencia escolar $^{4}$, de las cuales, veintitrés se limitan a reconocer solo la violencia entre iguales. (Véase Tabla 2). 
TABLA 2. ESFUERZOS LEGISLATIVOS EN RELACIÓN A LA VIOLENCIA ESCOLAR.

\begin{tabular}{|c|c|c|c|c|c|}
\hline Estado & $\begin{array}{l}\text { Cuenta } \\
\text { con Ley } \\
\text { específica } \\
\text { sobre } \\
\text { Violencia } \\
\text { Escolar }\end{array}$ & $\begin{array}{c}\text { Reconoce } \\
\text { la violencia } \\
\text { entre iguales }\end{array}$ & $\begin{array}{c}\text { Reconoce } \\
\text { la Violencia } \\
\text { Institucional }^{1}\end{array}$ & $\begin{array}{c}\text { Contempla } \\
\text { mecanismos de } \\
\text { intervención de } \\
\text { la violencia entre } \\
\text { iguales. }\end{array}$ & $\begin{array}{c}\text { Contempla } \\
\text { al Trabajador } \\
\text { Social para } \\
\text { Intervenir en } \\
\text { la Violencia } \\
\text { Escolar }\end{array}$ \\
\hline Aguascalientes & • & • & • & & \\
\hline Baja California & $\bullet$ & $\bullet$ & $\bullet$ & & \\
\hline $\begin{array}{l}\text { Baja California } \\
\text { Sur }\end{array}$ & $\bullet$ & $\bullet$ & & & \\
\hline Campeche & & & & $\bullet$ & \\
\hline Coahuila & $\bullet$ & $\bullet$ & & $\bullet$ & \\
\hline Colima & $\bullet$ & $\bullet$ & & $\bullet$ & \\
\hline \multicolumn{6}{|l|}{ Chiapas } \\
\hline Chihuahua & $\bullet$ & $\bullet$ & & $\bullet$ & \\
\hline Cdmx & $\bullet$ & $\bullet$ & & & \\
\hline Durango & & & & $\bullet$ & \\
\hline Guanajuato & $\bullet$ & $\bullet$ & & $\bullet$ & \\
\hline Guerrero & $\bullet$ & $\bullet$ & & & \\
\hline Hidalgo & $\bullet$ & $\bullet$ & & & \\
\hline Jalisco & & & & $\bullet$ & \\
\hline \multicolumn{6}{|l|}{ México } \\
\hline Michoacán & $\bullet$ & $\bullet$ & & $\bullet$ & \\
\hline Morelos & $\bullet$ & $\bullet$ & & & \\
\hline Nayarit & $\bullet$ & $\bullet$ & & $\bullet$ & \\
\hline Nuevo León & $\bullet$ & $\bullet$ & $\bullet$ & $\bullet$ & $\bullet$ \\
\hline Oaxaca & $\bullet$ & $\bullet$ & & $\bullet$ & \\
\hline Puebla & $\bullet$ & $\bullet$ & & $\bullet$ & \\
\hline \multicolumn{6}{|l|}{ Querétaro } \\
\hline Quintana Roo & $\bullet$ & $\bullet$ & & $\bullet$ & \\
\hline San Luis Potosí & $\bullet$ & $\bullet$ & & $\bullet$ & \\
\hline Sinaloa & $\bullet$ & $\bullet$ & & $\bullet$ & \\
\hline \multicolumn{6}{|l|}{ Sonora } \\
\hline \multicolumn{6}{|l|}{ Tabasco } \\
\hline Tamaulipas & $\bullet$ & $\bullet$ & & $\bullet$ & \\
\hline Tlaxcala & $\bullet$ & $\bullet$ & & $\bullet$ & \\
\hline Veracruz & $\bullet$ & $\bullet$ & & $\bullet$ & \\
\hline Yucatán & $\bullet$ & $\bullet$ & & $\bullet$ & \\
\hline Zacatecas & $\bullet$ & $\bullet$ & & $\bullet$ & \\
\hline
\end{tabular}

(Elaboración propia) 
En el caso de Nuevo León la Ley para Prevenir, Atender y Erradicar el Acoso y la Violencia Escolar en su artículo $3^{\circ}$ fracción XIX señala la violencia escolar como:

"La acción u omisión dolosa con la intención de dañar física o psicológicamente a una persona perteneciente a la comunidad educativa, ya sean alumnos, profesores, padres, personal directivo o subalterno y que se produce dentro de las instalaciones escolares, o bien en otros espacios directamente relacionados con el ámbito escolar, alrededores de la escuela o lugares donde se desarrollan actividades extraescolares. También, se considera violencia escolar las acciones que se realicen a través de las redes sociales, o cualquier tipo de comunicación, escrita, electrónica o a través de imágenes que pretenda dañar la dignidad de las personas en el ámbito señalado."
Siendo la definición más completa sobre violencia escolar que encontramos en las leyes revisadas para este estudio. Una singularidad de esta Ley es que establece la obligatoriedad para las escuelas de educación básica y media superior el contar con un psicólogo o trabajador social responsable de coadyuvar con las acciones relacionadas con la prevención, atención y erradicación del acoso y la violencia escolar. Sobre esta línea de ideas, en el Estado de Nuevo León, la Secretaría de Educación ofrece diferentes programas relacionados con la promoción de la convivencia escolar y el manejo de resolución de conflictos, sin embargo, dichos programas son orientados a la educación básica (Coordinación Estatal de Seguridad Escolar, 2016), excluyendo totalmente medidas dirigidas hacia el nivel medio superior. (Véase Tabla 3).

TABLA 3. PROGRAMAS DE LA SECRETARIA DE EDUCACIÓN DE NUEVO LEÓN SOBRE CONVIVENCIA ESCOLAR Y MANEJO DE CONFLICTOS

\begin{tabular}{|l|l|}
\hline \multicolumn{1}{|c|}{ PROGRAMA } & \multicolumn{1}{|c|}{ OBJETIVOS } \\
\hline Unidades de Asesoría Pedagógica (UAP) & $\begin{array}{l}\text { Brindar servicios de asesoría psicopedagógica } \\
\text { a las escuelas, maestros, padres de familia y } \\
\text { alumnado de educación básica que presente } \\
\text { problemas de aprendizaje, de conducta o } \\
\text { emocionales y situaciones de riesgo de las } \\
\text { distintas regiones del Estado. }\end{array}$ \\
\hline Acompáñame a crecer & $\begin{array}{l}\text { Desarrollar en alumnos de educación básica los } \\
\text { aspectos cognitivos, emocionales y axiológicos } \\
\text { necesarios para una convivencia adecuada } \\
\text { como parte de una sociedad. }\end{array}$ \\
\hline Conferencias para prevenir la violencia escolar & $\begin{array}{l}\text { Fortalecer por medio de ejemplos positivos } \\
\text { (alumnos de la UANL) la resolución pacífica de } \\
\text { conflictos, prevención de la violencia y proyecto } \\
\text { de vida claro, orientado a alumnos de primaria y } \\
\text { secundaria. }\end{array}$ \\
\hline
\end{tabular}


Capacitación en mediación escolar
Dar a conocer los antecedentes, características, elementos, funcionamiento y dinámica de la mediación como mecanismo para la resolución pacífica de conflictos. Los asistentes comprenderán la importancia, conocerán las estrategias y adquirirá habilidades y destrezas para el ejercicio de la mediación escolar. Asimismo, obtendrán conocimientos con relación a la enseñanza de la mediación en el requerido espacio. Dirigido a docentes y directivos de escuelas de educación básica.

(Elaboración propia)

Ante esta situación, decidimos realizar un estudio para conocer la incidencia de la violencia escolar en alumnos de una escuela del nivel medio superior en el Estado de Nuevo León.

\section{MATERIAL Y MÉTODOS}

El presente es un estudio exploratorio, para la obtención de la información se utilizó una escala de Likert con veintitrés reactivos, a una muestra aleatoria de 150 estudiantes de una población total de 1050 que se encuentran inscritos dentro del turno nocturno de la escuela preparatoria Emiliano Zapata. La aplicación del instrumento se realizó en el mes de marzo del 2020 a través de la plataforma en línea google forms. El cuestionario utilizado fue redactado con base en los estudios de Escalera (2018) y Del Tronco \& Madrigal (2013) teniendo como resultado los siguientes reactivos:

\section{Edad del Encuestado}

II. Sexo

1. ¿Te han insultado compañeros tuyos? (ofendiéndote o diciéndote apodos, etc.)

2. ¿Te ha pasado que uno o varios de tus compañeros te agredan físicamente? (con golpes, empujones etc.)
3. ¿Te ha pasado que uno o varios compañeros tuyos te amenazaran con hacerte daño?

4. $\quad i T e$ ha pasado que tus compañeros se burlaran de ti por alguna característica física tuya o por tu forma de ser? (por ejemplo, por tu peso, tu color de piel, la música que escuchas, etc.)

5. ¿Te ha pasado que quisiste participar en algún juego o actividad, pero no pudiste porque tus compañeros no te dejaron?

6. ¿Has hecho algo que no querías hacer debido a la presión o amenaza de uno o varios de tus compañeros?

7. ¿Algún profesor tuyo te ha agredido físicamente? (golpeándote o empujándote, etc.)

8. ¿Algún profesor tuyo te ha insultado?

9. ¿Algún profesor tuyo te ha amenazado con hacerte daño?

10. ¿Algún profesor te ha invitado a salir?

11. ¿Has sentido miedo cuando has estado en presencia de algún profesor?

12. ¿Algún profesor te ha agredido, molestado o burlado de ti por tu lugar de origen? 
13. ¿Algún profesor ha ejercido su autoridad para calificarte mal?

14. ¿Algún profesor te ha agredido, molestado o se ha burlado de ti por tu apariencia? (forma de vestir, de corte de cabello, etc.)

15. ¿Algún profesor te ha agredido, molestado o se ha burlado de ti por tu orientación sexual?

16. ¿Algún profesor te ha pedido dinero a cambio de una calificación?

17. ¿Te has quejado formalmente de algún profesor?

18. ¿Te has quejado formalmente de algún profesor $y$ tu queja no fue atendida?

19. ¿Después de quejarte formalmente de algún profesor, las autoridades escolares hicieron algo al respecto

20. ¿Te han robado dentro de la escuela?

21. ¿Has sido agredido por uno o varios compañeros tuyos con armas? (cuchillo, pistola, etc)

\section{CONSIDERACIONES ÉTICAS}

Al momento de contestar la encuesta se les hace saber a las estudiantes que la información que proporcionan es de carácter confidencial y sería utilizada para la realización de esta investigación. Los resultados arrojados fueron capturados en una base de datos y fueron procesados por el software estadístico SPSS.

\section{RESULTADOS Y DISCUSIÓN}

De acuerdo a Arias y Amador (2019) las dimensiones de la violencia escolar hacen alusión a tres categorías: 1) la violencia entre pares, relacionada a circunstancias dañinas para una persona que son resultado de la interacción entre los mismos estudiantes (bullying); 2) la violencia institucional, que se refiere a imperativos de poder personal directivo y académico hacia los alumnos, y 3 ) la violencia del entorno hacia la escuela, que involucra las problemáticas exteriores que afectan colateralmente. A continuación se presentan los resultados de las encuestas contestadas por los estudiantes de preparatoria, organizados a partir las dimensiones anteriormente señaladas.

\section{VIOLENCIA ENTRE PARES}

Podemos observar que aproximadamente el $70 \%$ de los estudiantes que respondieron la encuesta manifestaron haber sido insultados en clase; el $16.6 \%$ señalo que fue agredido por alguno de sus compañeros y el $26.6 \%$ menciono haber sido amenazado al menos una vez durante el tiempo de clases como lo podemos ver en la gráfica (001). Según datos de la organización Bullying Sin Fronteras, México se encuentra entre los 20 países que registran mayor índice de casos registrados relacionadas al acoso escolar; esta organización menciona que solo 2 de cada 10 agredidos reportan el suceso (Rodríguez,2018). 


\section{Gráfica 001}

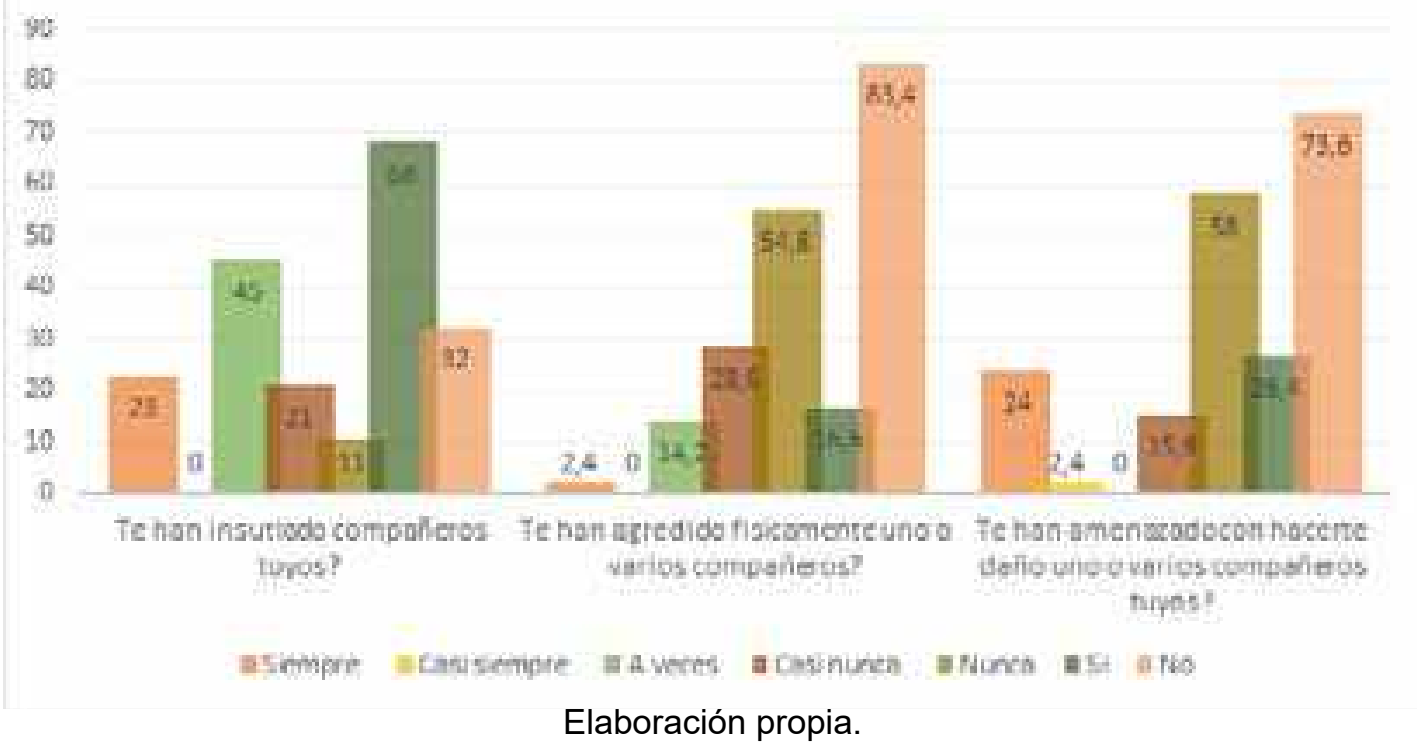

Cuando se habla de acoso escolar las agresiones psicológicas tienen un papel importante, dentro de ellas se encuentran las burlas por alguna característica, el lugar de origen, entre otros; estas acciones traen como consecuencia baja autoestima, puesto que se denigra su dignidad personal que se realizan para que el agresor tenga dominio y control sobre el otro (Gracia y Freyre, 2008). El $58 \%$ de los sujetos que respondieron la encuesta han sido insultados por una característica física o por su forma de ser; EL 28\% menciono haber sido excluido por sus compañeros al no permitirle su participación en alguna actividad o juego como lo podemos ver en la gráfica (0o2).

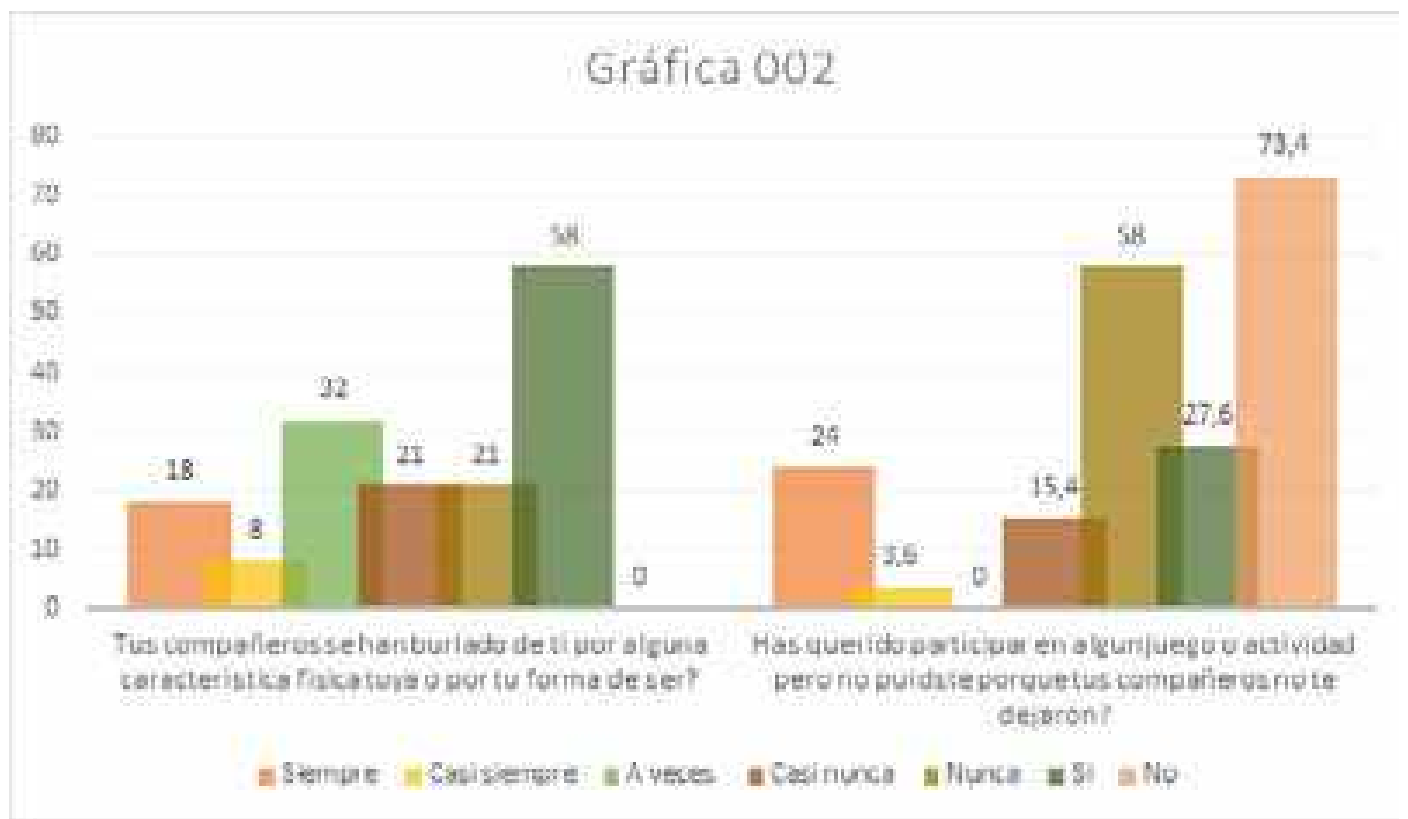

Elaboración propia 
Dentro de los tipos de violencia escolar también se encuentran la agresión social, que va de la mano con la agresión psicológica. En este estudio el $21 \%$ de los estudiantes indicaron haber realizado alguna acción que no eran de su agrado por presión o amenaza de sus compañeros de clase como podemos ver en la gráfica 003.

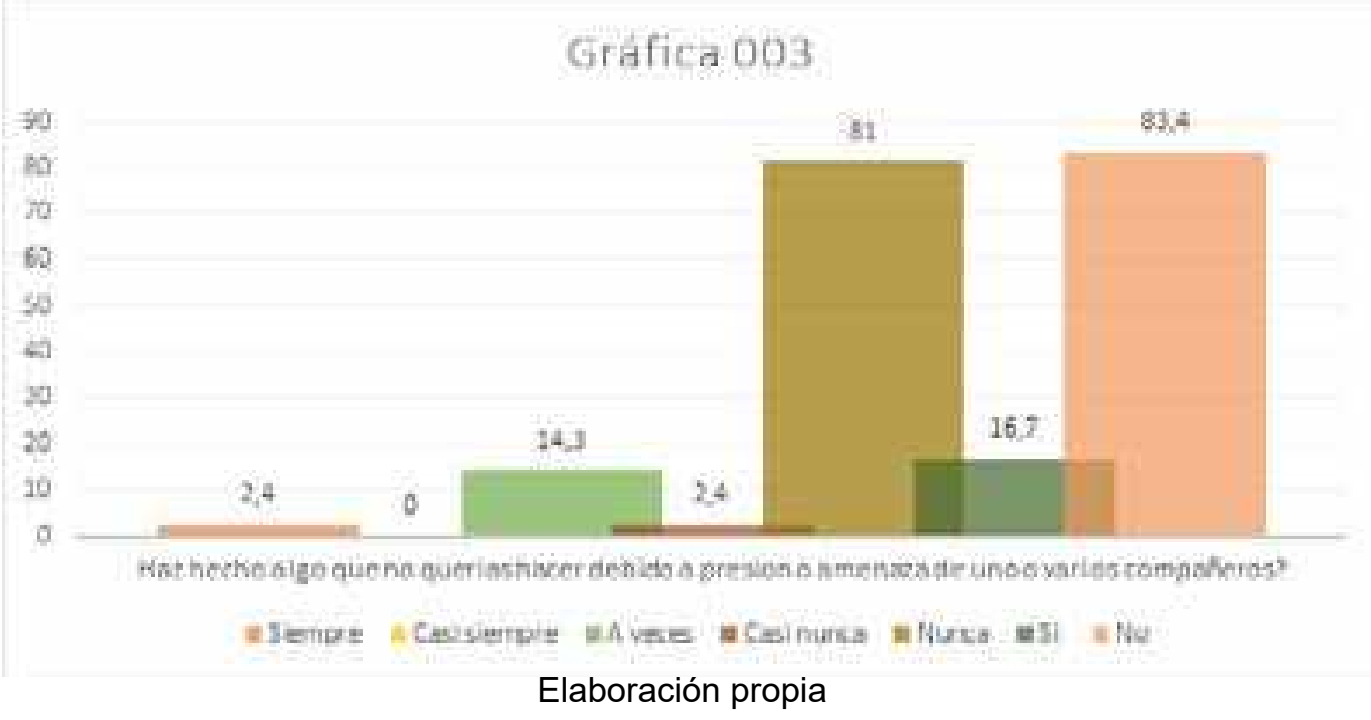

\section{VIOLENCIA INSTITUCIONAL}

Las instituciones (en este caso los contextos escolares), son esferas donde se generan sentidos y formas de organización similares a los de la familia, además son espacios de necesidades e imposiciones sociales que demandas respuestas y una adaptación continua a coerciones y normas formales. Actualmente la violencia institucional es considerada una problemática que solo comprende aspectos culturales, económicos y políticos bajo una estructura organizacional a base de poder, conflicto, liderazgo, toma de decisiones, comunicación y/o cambios (Barrientos, 2015).
En los espacios escolares la violencia institucional es por lo general ejercida por los profesores, que son los que pasan mayor tiempo con los estudiantes por actividades propias del proceso de enseñanza aprendizaje. Dentro del contexto en el que se realizó este estudio ningún estudiante señalo haber sido agredido físicamente por algún profesor, pero sí haber sido insultado $(24.3 \%)$ y recibido amenazas (14\%). El $16.7 \%$ de los estudiantes que respondieron la encuesta mencionaron haber sido invitados a salir por algún profesor, de este porcentaje la tercera parte son mujeres, lo que podría indicarnos presencia de violencia sexual en el contexto educativo como lo podemos ven en la gráfica (grafica 004). 


\section{Gráfica 004}

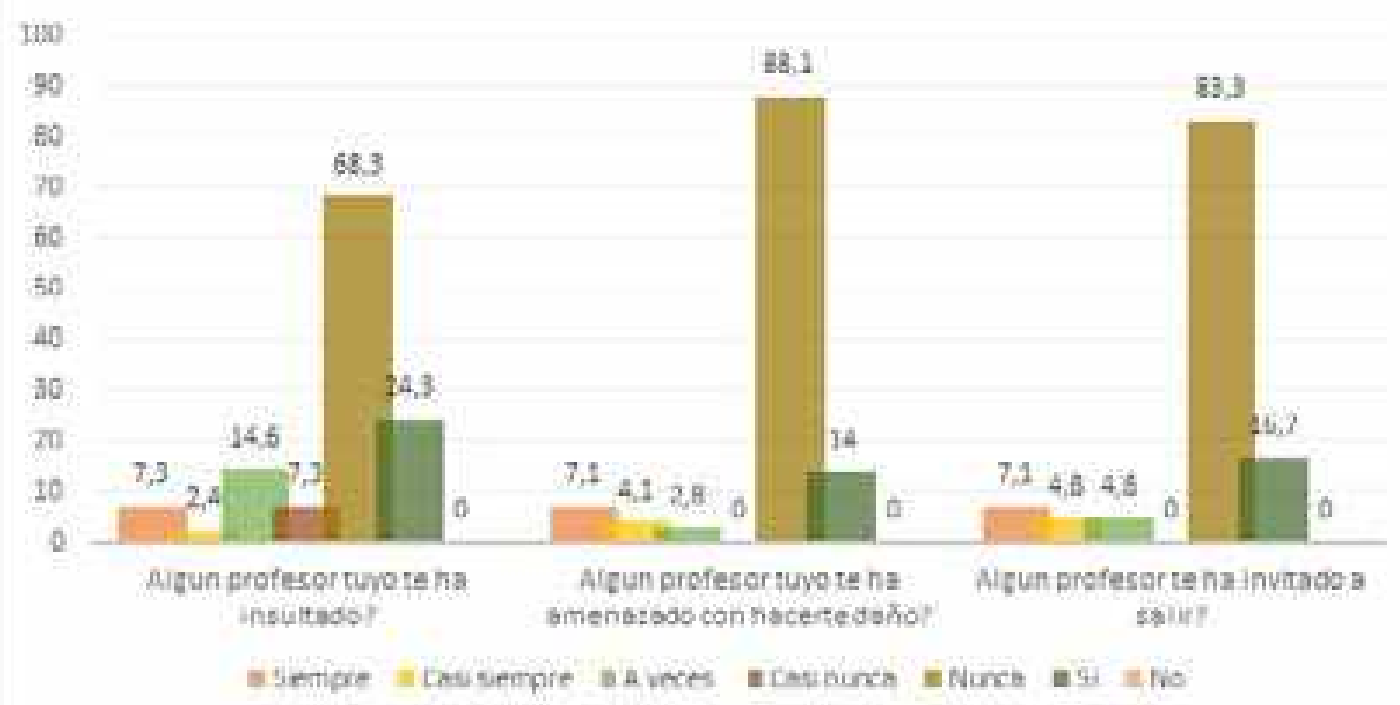

\section{Elaboracion propia}

Siguiendo a Treviño y Chávez (2017) la violencia institucional se realiza a base de la posición de poder del profesorado, quién al dejar de lado sus principios de ética profesional y de responsabilidad social que adquiere con su formación, opta por excluir, maltratar o afectar el plan de vida del alumnado
El $9 \%$ de los estudiantes menciono haber sentido miedo al estar en presencia de algún profesor, el $17 \%$ de índico que al menos alguna vez un profesor le pidió dinero a cambio de una calificación y el $26 \%$ menciono que algún profesor ejerció su autoridad para calificarle de forma errónea (grafica 005).

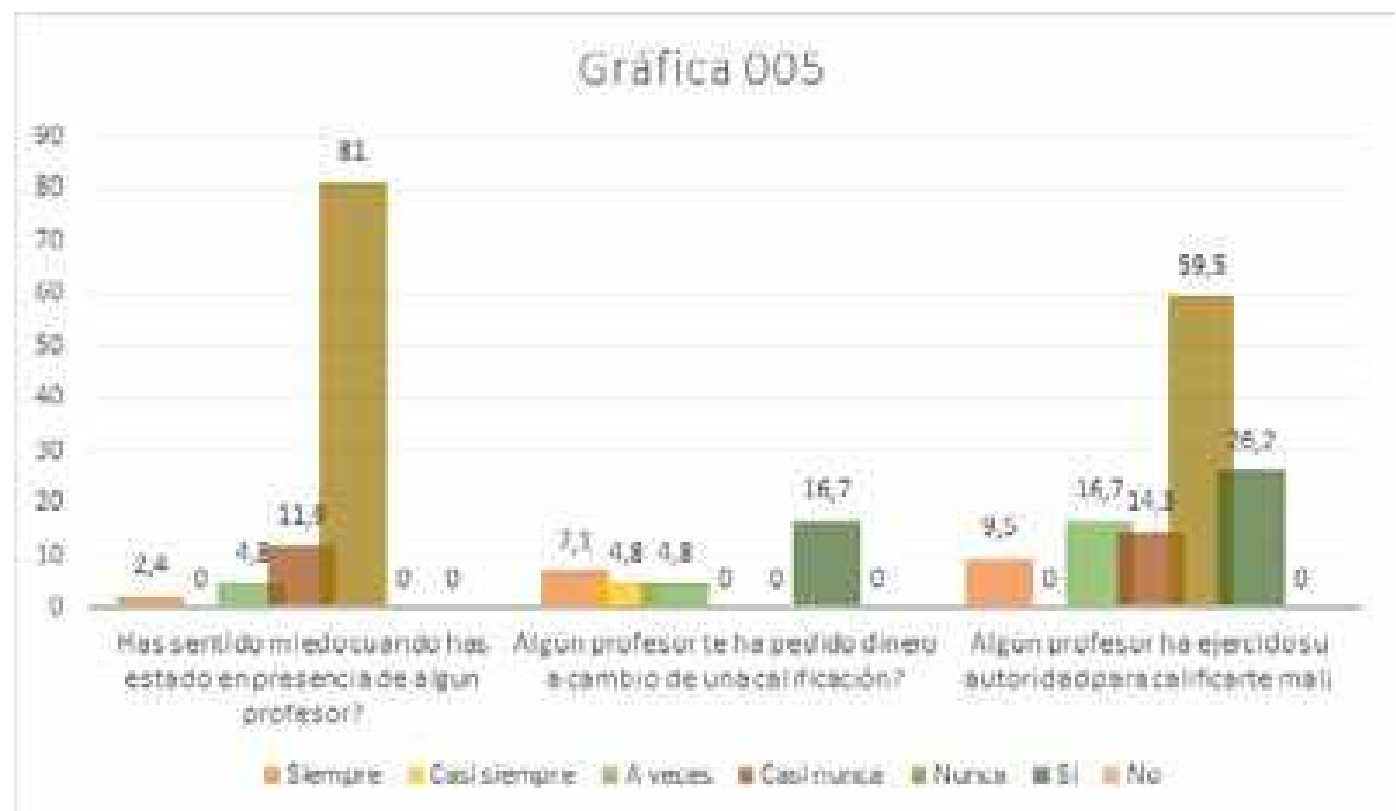

Elaboración propia 
En Nuevo León Cervantes (2013) realizó una investigación en contextos universitarios sobre los distintos tipos de violencia, entre los que destacó la institucional. Los datos de dicha investigación variaron entre $7.4 \%$ y $13.1 \%$ de conductas violentas del profesor hacia el alumno; estos resultados coinciden con los encontrado en este estudio; el $12 \%$ de los estudiantes mencionaron haber sido agredidos, molestado o burlado por su apariencia, el $14 \%$ por su orientación sexual (grafica 006).

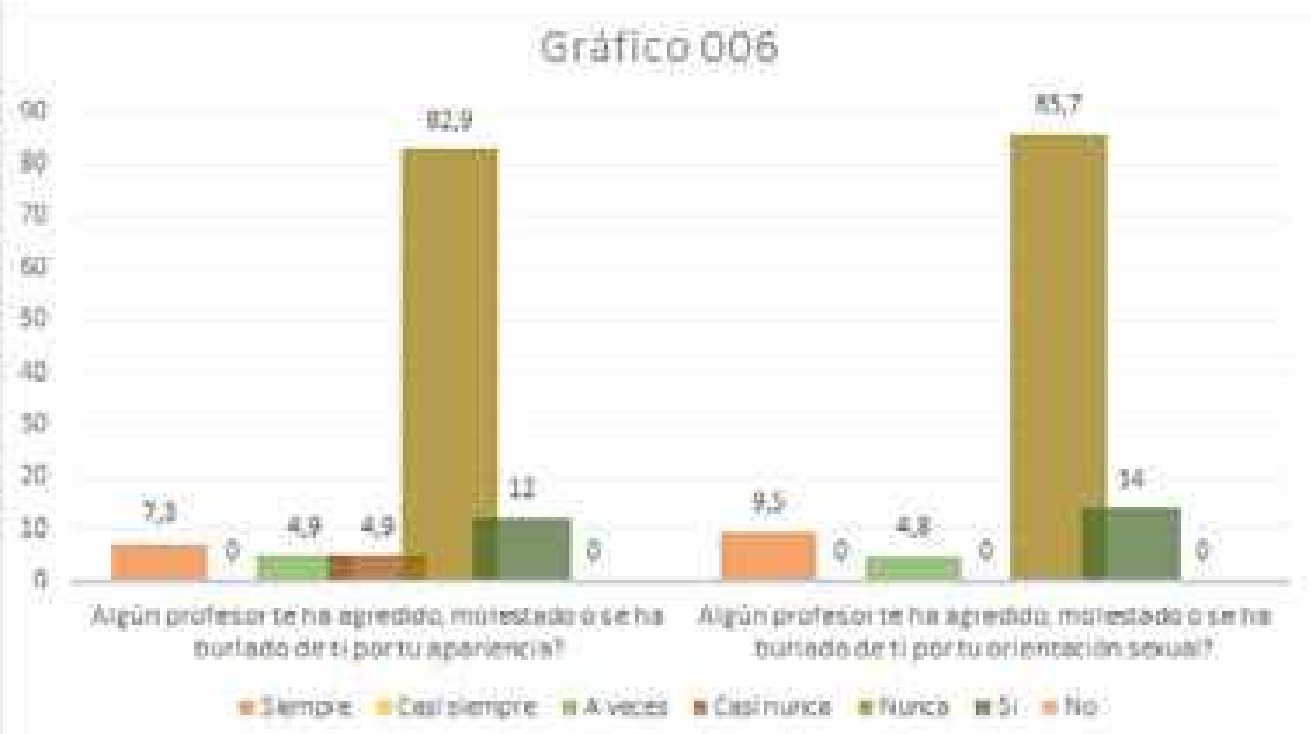

Elaboracion propia

Las conductas y prácticas violentas en los contextos escolares son variadas, comprenden desde el uso de motes, hasta actitudes de indiferencia y discriminación a los alumnos, también incluyen practicas 0 normas comportamentales de los directivos dañan la integridad a estos (Del Tronco y Madrigal, 2013).
El $19 \%$ de los estudiantes de la preparatoria en donde se realizó este estudio mencionaron presentar formalmente una queja de algún profesor; de este porcentaje15\% afirma que su queja fue atendida y el $20 \%$ que las autoridades hicieron algo al respecto.

\section{Grafica 007}

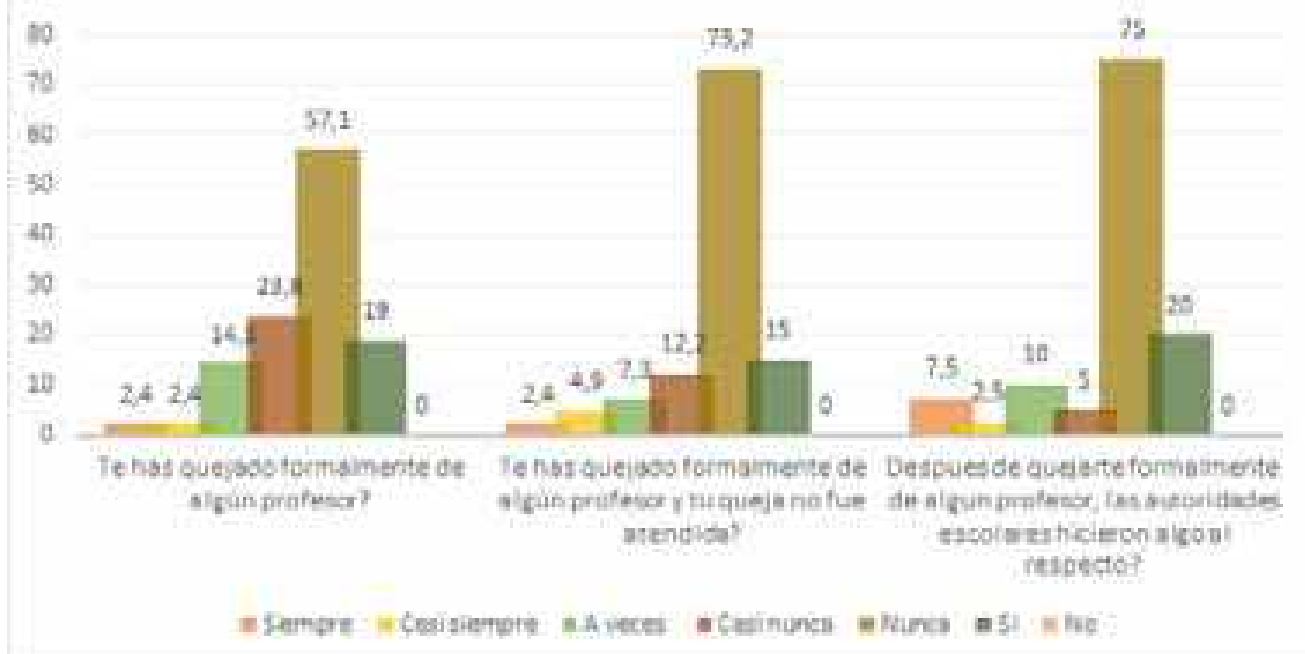

Elaboración propia 


\section{VIOLENCIA DEL ENTORNO HACIA LA ESCUELA}

También se encontró violencia de contexto entre los estudiantes encuestados, el $17 \%$ afirmo que le han robado dentro de la escuela, lo cual resulta significativo porque los mismos alumnos los que generan inseguridad y acciones violentas al interior de las aulas, así mismo, aunque poco significativo estadísticamente pero sí relevante desde el punto de vista social, el $2.4 \%$ menciono haber sido agredido por uno o varios de sus compañeros con algún tipo de arma.

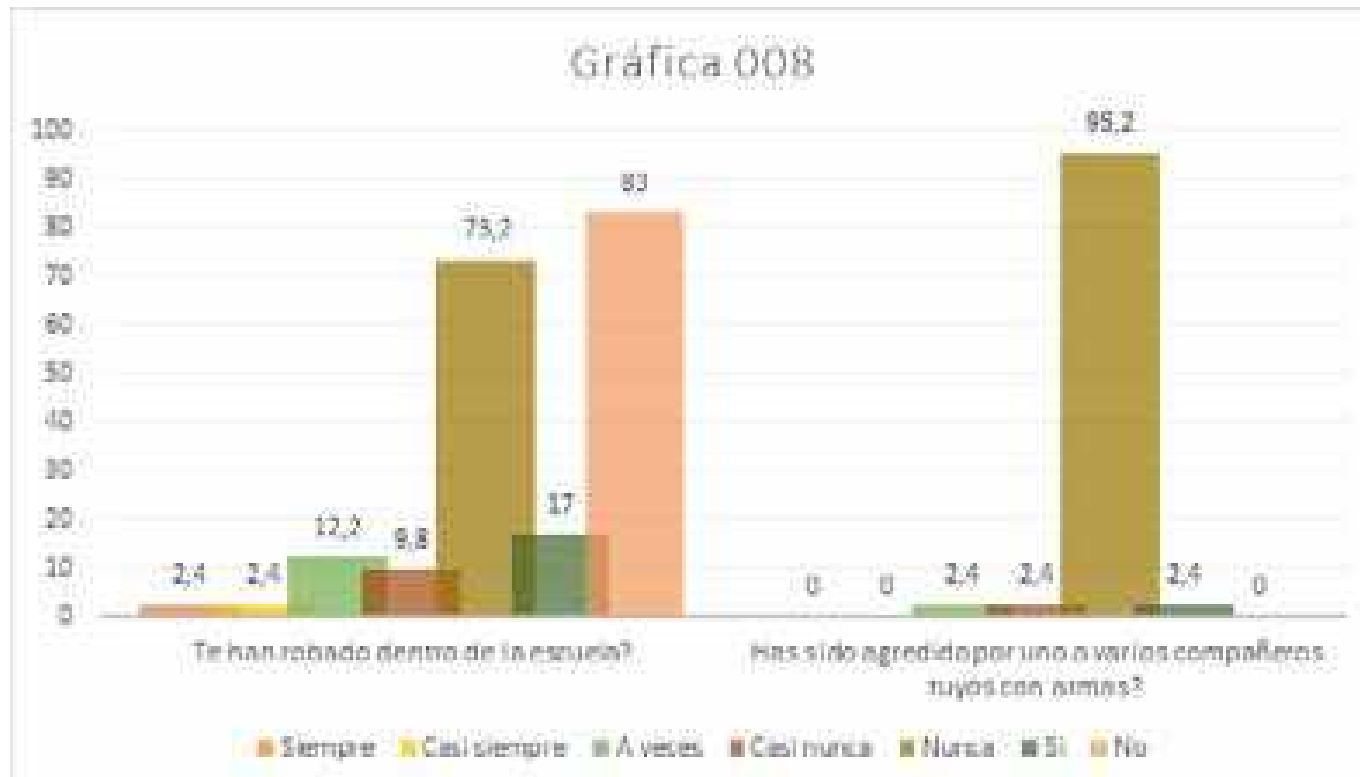

Elaboración propia

\section{CONCLUSIONES}

Dentro de la discusión sobre la violencia escolar existen diferentes maneras o formas de definir la problemática, en algunos contextos se llama acoso escolar (bullying), cuando es entre pares o compañeros; y acoso académico cuando es de autoridades o docentes hacia los alumnos o personas subordinadas. No obstante para fines de este estudio entendemos la violencia escolar como: violencia entre pares y violencia institucional; centrando la atención de este estudio a la violencia institucional que abordaremos y conceptualizaremos como hostigamiento académico.

En este artículo se logró destacar diversos aspectos que se relacionan directamente con la violencia institucional en el contexto escolar.

La información que se recabó fue mediante un cuestionario en línea el cual arrojó que; al menos el $70 \%$ de los alumnos ha sido insultado por sus compañeros, así como también han sido agredidos física y verbalmente, han vivido experiencias de exclusión o de presión para realizar alguna actividad.

En cuanto a la relación alumno- profesor se observó que el $24.3 \%$ de los encuestados respondieron haber estado en situaciones en las que se enfrentaron a insultos mientras que el $14 \%$ ha sido víctima de amenazas, y el $16 \%$ fueron invitados a salir con algún profesor.

Por otra parte, en cuanto al aprovechamiento del poder del profesorado, algunos de los alumnos expresaron haber sentido miedo con la presencia de su maestro, otros indicaron que han experimentado que un maestro le pida dinero a cambio de una calificación, así como también se detectó que un porcentaje considerable dio a conocer por medio de las encuestas que en 
algunas ocasiones se ha ejercido autoridad para calificar de manera negativa.

En cuanto a las agresiones profesor- alumno se descubrió que una gran parte de los estudiantes los han molestado por su apariencia y orientación sexual.

Respecto a las quejas de presentadas por parte de los alumnos hacia sus profesores, se percibió que el $19 \%$ ha mostrado interés en denunciar acciones que los afecten como estudiantes de los cuales se destacó que no todas fueron atendidas, más sin embargo en unas ocasiones las autoridades si hicieron algo al respecto.

Finalmente se logró observar que la inseguridad dentro de los planteles educativos está al orden del día ya que se expresó que un número significativo de alumnos respondió haber sido robado dentro de la escuela, y por otro lado la mayoría de los encuestados afirmó nunca haber sido asaltado por compañeros con armas.

\section{CONTRIBUCIÓN DESDE EL TRABAJO SOCIAL A ERRADICACIÓN DE LA VIOLENCIA INSTITUCIONAL EN EL CONTEXTO ESCOLAR.}

La intervención llevada a cabo desde la perspectiva del Trabajo Social consta de cinco fases interrelacionadas. El proceso puede llevarse a cabo tanto con individuos, familias o comunidades, el cual comienza con la observación, continúa con la interpretaciónvaloración, diseño de plan de acción, acción y evaluación.

La intervención es el mismo proceso en todos los casos, sin embargo se diferencia de cada situación dependiendo de dónde provenga la demanda, ya sea del alumno, profesorado o padres.

Áviles \& Monjas (2006) afirman que para prevenir el maltrato es necesario cambiar las actitudes, valores y conductas que estén fomentando la violencia en el contexto escolar, así como también es preciso introducir cambios relacionados con la convivencia implicando la relación maestrosalumnos.

Respecto a la contribución a la erradicación de la violencia institucional, los trabajadores sociales proponen: la política anti- bullying, desarrollo de programas de educación para la convivencia y prevención de la violencia y maltrato entre iguales en el curricular escolar. En las cuales se integrará lo social en lo educativo para conseguir relaciones de solidaridad grupal entre personas que participen en procesos preventivos.

\section{REFERENCIAS BIBLIOGRAFICAS}

Arias, Y., y Amador, S. (2019). Acoso escolar a estudiantes de una institución de educación superior en Nuevo León. En M. Castro (Presidente), Congreso Nacional e Internacional de Investigación en Trabajo Social. Redes Temáticas; Dilemas y Perspectivas. Ponencia llevada a cabo en Universidad Juárez del Estado de Durango.

Barrientos, P. (2015). Violencia institucional: hacia un nuevo enfoque. https://www.researchgate.net/ publication/289980382

Bauman, Z. (2008). Múltiples culturas, una sola humanidad. Buenos Aires: Katz Editores.

Berger, P., \& Luckmann, T. (1968). La construcción social de la realidad. Madrid: Amorrortu Editores.

Calmaestra, J. (2011). Cyberbullying: prevalencia y características de un nuevo tipo de bullying indirecto. Córdoba: Universidad de Córdoba. 
Cepeda, E., Pacheco, P., García, L., \& Piraquive, C. (2008). Acoso escolar a estudiantes de educación básica a media. Revista de salud pública, 517-529.

Cervantes, M., Sánchez, C., y Villalobos, M. (2014). Percepción de la violencia del docente hacia el alumno en instituciones de educación superior. Red de Investigación Educativa UANL, (1), 378-392. http://www. reddeinvestigacioneducativa.uanl.mx/ wp-content/uploads/publicaciones/ rie_2014.pdf

Cobo, P., \& Tello, R. (2008). Bullying en México. México: Editorial Lectorum.

Coordinación Estatal de Seguridad Escolar. (10 de Marzo de 2016). Secretaria de Educación del Estado de Nuevo León. Obtenido de http://www.nl.gob. $\mathrm{mx} / \mathrm{publicaciones/programas-de-}$ prevencion-y-servicios-de-atencion

Craig, W., \& Peppler, D. (2007). Undestending bullying: From research to practice. Canadian Psychology, 86-93.

Del Tronco, J., \& Madrigal, A. (2013). Violencia Escolar en México: una exploración de sus dimensiones y consecuencias. Trabajo Social UNAM, 24-43.

Escalera Silva, L. A. (18 de diciembre de 2018). Análisis de la Violencia Cibernetica en el Contexto Escolar en las Preparatorias de la Universidad Autónoma de Nuevo León, del Área Metropolitana de Monterrey. Ciudad Universitaria: Tesis Doctoral, Universidad Autónoma de Nuevo León.

Escalera, L., Silva, L., \& Contreras, A. (2019). El derecho a una escuela libre de violencia. Esfuerzos legislativos de las entidades federativas dirigidos a combatir la violencia escolar. Realidades, 95-121.

Estevez, E. (2005). Violencia, victimización y rechazo escolar en la adolescencia. Valencia: Publicaciones Universidad de Valencia.

Garaigordobil, M. (2013). Cyberbullying. Screening de acoso entre iguales. Madrid: TEA.

Gergen , K., \& Gergen, M. (2011). Reflexiones sobre construcción social. Barcelona: Paidós.

Harries, S. y. (2003). El acoso en la escuela. Los agresores, las víctimas y los espectadores. México: Paidós.

Olewus, D. (1998). Conductas de acoso y amenazas entre escolares. Madrid: Morata.

Olewus, D. (2005). Bullying en la escuela: datos e intervención. En J. Sanmartín, Violencia y Escuela (págs. 13-30). Valencia: Centro Reina Sofía para el estudio de la violencia.

Olewus, D. (2012). Cyberbullying: an overrated phenomenon? European journal of developmental psychology, 520-538.

Organización de las Naciones Unidas para la Educación, la Ciencia y la Cultura. (2019). Behind the numbers: Ending school violence and bullying. Recuperado de https://unesdoc.unesco. org/ark:/48223/pf0000366483

Rodríguez, P. A. (2018). Bullying en México: Estadística, Casos, Asociaciones y Cómo Actuar. Recuperado de https:// www.lifeder.com/bullying-mexico/ 
Treviño, M., De la Cruz, M., Chávez, G., y Hauad, I. (2013). Violencia entre iguales en el campus del área médica de la Universidad Autónoma de Nuevo León. Recuperado de http://eprints.uanl. mx/8065/1/d1_3.pdf

\section{(Footnotes)}

1 Reconoce la posible existencia de la relación de poder y subordinación entre alumno y docente/autoridad educativa, sin denominarla violencia institucional. 